\title{
Sind Wirbelsäulenleiden genetisch determiniert?
}

Das dänische Zwillingsregister ist eine schier unerschöpfliche Datenquelle, wenn es darum geht, genetische Faktoren für bestimmte Erkrankungen aufzudecken. So gibt das Register etwa auch genau Auskunft über Auftreten und Lokalisation von Wirbelsäulenleiden bei Zwillingen. Aus diesen Daten kann abgeleitet werden, zu welchem Prozentsatz die Beschwerden genetisch bedingt sind.

- Dänische Forscher werteten die entsprechenden Daten von 15328 Zwillinge (Alter 20-71 Jahren) aus. 44\% waren monozygote und $56 \%$ dizygote Zwillinge.

Wie die Analyse zeigt, können 38\% aller lumbalen, $32 \%$ aller thorakalen und 39\% aller zervikalen Wirbelsäulenbeschwerden durch genetische Faktoren erklärt werden. Diese Daten, so meinen die Autoren, weisen auf eine gemeinsame genetische Basis vieler Schmerzsyndrome der Wirbelsäule hin.
KOMMENTAR: Die Ergebnisse sind im Zusammenhang mit anderen Befunden zu sehen, die implizieren, dass Wirbelsäulenbeschwerden nicht spezifische, lokal bedingte Entitäten, sondern Teil eines generellen Schmerzsyndroms sind. Nach den dänischen Daten scheinen dabei genetische Faktoren eine erhebliche Rolle zu spielen. Das würde vielleicht auch erklären, weswegen wir solchen Schmerzen häufig relativ hilflos gegenüberstehen. Die therapeutischen Konsequenzen dieser Ergebnisse sind noch schwer abzusehen. Sicher ist jedoch, dass sich hier ein Umdenken abzeichnet, was unser Verständnis bezüglich sogenannter "mechanischer" Wirbelsäulenleiden ändern wird.

E. ERNST $\equiv$

\begin{abstract}
-J. Hartvigsen et al.
Heritability of spinal pain and consequences of spinal pain: a comprehensive genetic epidemiologic analysis using a population-based sample of 15,328 twins ages 20-71 years. Arthritis Rheum. 61 (2009) 1343-1351
\end{abstract}

Genetische Komponente

Eltern mit Alzheimer

- Kinder gefährdet?

Für Deutschland werden 700 ooo Alzheimerkranke geschätzt. Es wird davon ausgegangen, dass der $M$. Alzheimer eine komplexe Krankheit mit einer starken genetischen Komponente ist. Eine Amyloidpathologie, Gefäßfaktoren und Entzündung sollen an der Pathogenese beteiligt sein. $\mathrm{Zu}$ den Ursachen ist weniger bekannt. Bei Blutuntersuchungen fanden Forscher des Medical Center in Amsterdam (Niederlande) neue Hinweise, die für eine bessere Diagnostik genutzt werden sollen.

- Blutparameter von 206 noch gesunden Kindern, deren 92 Eltern an Alzheimer erkrankt waren, wurden mit denen von 200 Kindern aus 97 gesunden Familien verglichen. Bestimmt wurden der APOE-4-Genotyp, die Blutdruckwerte, die Zytokinproduktion von Blutzellen nach LPS-Stimulation in vitro und zirkulierende Entzündungsmarker.

Die Kinder von Alzheimerpatienten trugen das APOE-4-Gen. das als potenzieller Krankheitsauslöser diskutiert wird. viel häufiger ( $47 \%$ versus $21 \%, p<0,001)$. Sie hatten höhere systolische $(p=0,006)$ und diastolische ( $p<0,001)$ Blutdruckwerte. Ihre Blutzellen bildeten in vitro mehr proinflammatorische Zytokine $(p<0,001$ bis $p=0,04)$. Diese Assoziationen waren vom APOE-4Genotyp unabhängig.

KOMMENTAR: Bluthochdruck und die Expression eines typischen, proinflammatorischen Zytokinprofils im mittleren Lebensalter sind möglicherweise frühe Risikoindikatoren für die Alzheimererkrankung, die sich für Screeninguntersuchungen eignen.

\section{K. MALBERG =}

-E. van Exel et al.

Vascular factors and markers of inflammation in offspring with a parental history of late-onset Alzheimer Disease. Arch. Gen. Psychiatry 66 (2009) 1263-1270 $\checkmark$ Je nach Lokalisation haben Rückenschmerzen eine starke genetische Basis.

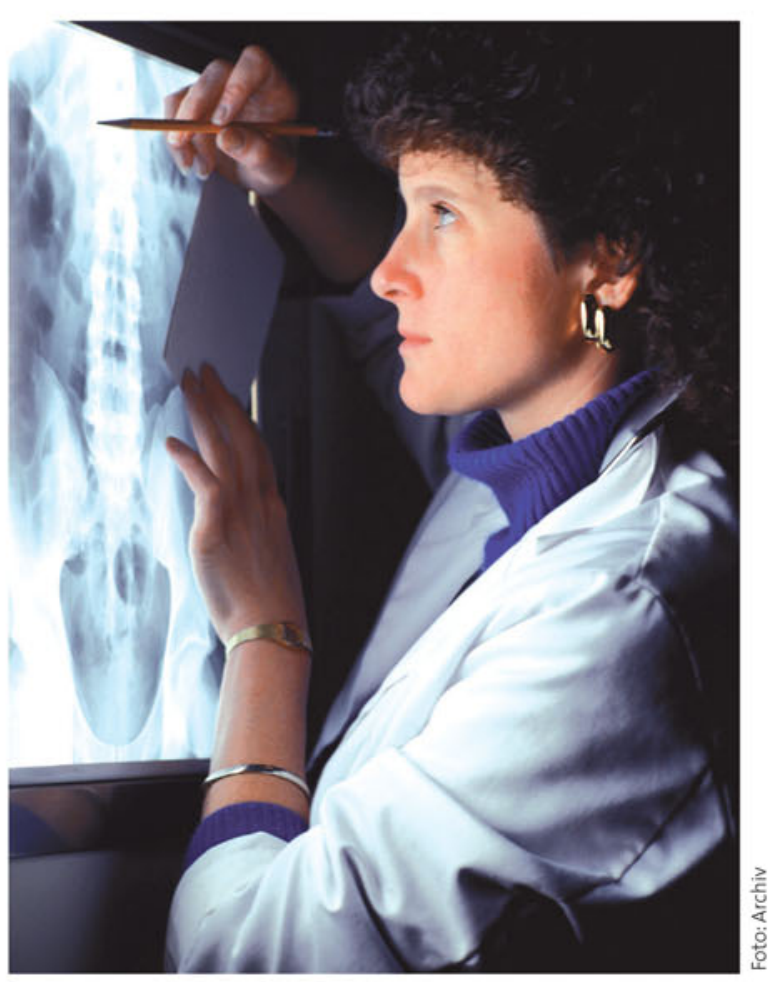

